\title{
Choroidal Neovascularization Regression on Fluorescein Angiography after VEGF Blockade
}

\author{
Diana V. Do Lark Greenwald Mohamed Ibrahim \\ Yasir Sepah Quan Dong Nguyen \\ Wilmer Eye Institute, Johns Hopkins Hospital, Baltimore, Md., USA
}

\section{Key Words}

Choroidal neovascularization · Fluorescein angiography · Age-related maculopathies

\begin{abstract}
Background: Intravitreal vascular endothelial growth factor (VEGF) inhibitors stabilize vision in a majority of patients with neovascular age-related macular degeneration (AMD) and can improve vision in almost $40 \%$ of patients. However, some individuals who respond to antiVEGF treatment still lose vision due to the formation of geographic atrophy (GA). While optical coherence tomography is often the primary imaging modality used, fluorescein angiography (FA) can provide useful information on GA development after choroidal neovascularization (CNV) regression.
\end{abstract}

Methods: A retrospective chart review was conducted to evaluate the changes seen on FA over a 47-month period for 3 patients with neovascular AMD treated with anti-VEGF inhibitors.

Results: All 3 patients were initially noted to have subfoveal CNV due to AMD at baseline; they were followed up monthly and treated on an as needed basis for at least 47 months with intravitreal VEGF inhibitors. All subjects had regression of their CNV lesions after VEGF blockade. Two subjects developed foveal atrophy.

Conclusions: This case series depicts the changes on FA seen over a 4-year period and shows that GA can occur with regression of CNV after treatment with VEGF inhibitors.

\section{Introduction}

Intravitreal vascular endothelial growth factor (VEGF) inhibitors are the standard treatment for subfoveal choroidal neovascularization (CNV) due to age-related macular degeneration (AMD) $[1,2]$. Though the original dosing regimen studied involved 
monthly injections, recently it has been observed that monthly follow-up with as needed treatment decreased the number of injections without sacrificing the gain in visual acuity [3-5]. Currently, most retina specialists employ optical coherence tomography (OCT) to evaluate CNV lesions and determine if additional VEGF inhibition is needed. Although OCT has become the mainstay for retinal imaging, fluorescein angiography (FA) is still important in the diagnosis and monitoring of retinal pathologies such as CNV, especially when geographic atrophy (GA) develops after CNV regression $[6,7]$.

We conducted a retrospective study to evaluate the dynamic changes seen on FA in 3 patients who had undergone serial FA imaging over 48 months of treatment with intravitreal (IVT) VEGF inhibitors for neovascular AMD.

\section{Materials and Methods}

This study was approved by the Johns Hopkins Institutional Review Board, which granted a waiver of consent for access to medical records for all persons included in the study. Retrospective chart review was performed for patients who had routine fluorescein angiograms and OCT performed as they were being treated with intravitreal VEGF inhibitors for neovascular AMD. Three sample cases are described below.

\section{Case Reports}

Case 1

Patient 1 is a 75 -year-old man who presented with decreased vision. Initially, his visual acuity was found to be 20/63 in the left eye with subfoveal CNV due to AMD. Baseline FA revealed a small subretinal hemorrhage and leakage from classic CNV (fig. 1a). One month after receiving an IVT injection with ranibizumab (RBZ), the CNV lesion decreased in size (fig. 1b), and it continued to regress and show decreasing amounts of leakage at months 3 and 6 , stabilized at month 9, and increased in leakage at month 12 (fig. 1c-f) as the patient received additional RBZ on an as needed basis depending on CNV activity seen on either FA and/or OCT. At month 47 and after a total of 16 ranibizumab injections, the CNV had completely regressed. However, GA had developed over the fovea resulting in a visual acuity (VA) of 20/250 (fig. 1g).

Case 2

Patient 2 is an 85-year-old man who presented with a VA of 20/80 in his right eye and subfoveal occult CNV due to AMD. On presentation, the CNV lesion was greater than 9 disc areas. One month after treatment with ranibizumab, the CNV had regressed and there was decreased leakage on FA. There was continued CNV regression through months 3, 6, 9 and 12 as the patient received additional RBZ on an as needed basis. At month 48 and after a total of 23 RBZ injections, there was no CNV leakage remaining, and the VA was 20/50, but some extrafoveal GA had developed.

Case 3

Patient 3 is an 80 -year-old woman who presented with a VA of 20/125 and subfoveal occult CNV due to AMD in the left eye. At the initial visit, there was subretinal hemorrhage and leakage from CNV (fig. 2a). After 2 RBZ treatments, there was regression of the CNV lesion at month 2 (fig. 2b), but leakage returned through month 12 (fig. 2c-e). This patient was followed up for a total of 57 months, during which she received $20 \mathrm{RBZ}$ injections and 2 bevacizumab injections. At her most recent visit, there was foveal GA with no CNV leakage, and her VA was 20/125 (fig. 2f). 


\section{Discussion}

Both FA and OCT images are able to monitor CNV activity after VEGF inhibition [8]. However, despite OCT being more commonly used today to monitor CNV lesions, FA and OCT have different benefits and limitations. OCT allows for visualization of the retina to document intraretinal or subretinal fluid and elevation of the retinal pigment epithelium (RPE) associated with CNV. FA visualizes the actual extent and size of the CNV lesion and documents leakage associated with it. Although spectral domain- (SD) OCT can sample a large area of the retina with high resolution, it still cannot document the extent of CNV seen on FA [9]. FA can truly document both the size of the CNV lesion and regression of it after treatment with VEGF inhibition, and aid in the diagnosis of geographic atrophy. More recently, ophthalmologists have also been employing SD-OCT for identifying areas of geographic atrophy that could be responsible for poor visual outcomes after anti-VEGF therapy.

Few studies have described long-term changes in CNV lesions with FA after VEGF inhibition. This case series documents $\mathrm{CNV}$ regression induced by anti-VEGF therapy. In addition, it documents the development of atrophy in the macula after CNV regression. The GA may be due to the natural history of the disease or it may be accelerated by CNV regression by anti-VEGF therapy. Rosenfeld et al. [10] evaluated causes of vision loss after anti-VEGF therapy and also demonstrated that RPE changes and atrophy were common causes of vision loss after long-term use of RBZ for neovascular AMD. Their study evaluated 71 eyes that lost at least 15 letters of VA over a 24-month period, and they found that RPE atrophy and/or abnormalities were the most common causes of visual loss among these patients. Additional studies of OCT and FA would be helpful to identify if chronic anti-VEGF therapy increases the risk of GA. In addition to OCT, FA is still an important tool for monitoring CNV activity after VEGF inhibition and for documenting $\mathrm{CNV}$ regression and the development of atrophy in the macula.

\section{Acknowledgement}

We would like to thank Shawn Wilker, MD, Assistant Professor of Ophthalmology at Case Western University, for his assistance with this project.

\section{Disclosure Statement}

Dr. D. Do has received research funding from Genentech, Regeneron and Heidelberg. Dr. Q.D. Nguyen has received research funding from Genentech, Regeneron, Heidelberg and Santen. He also serves as a consultant to Bausch and Lomb, and Santen. The remaining authors declare no conflict of interest.

This work has not been previously presented. 


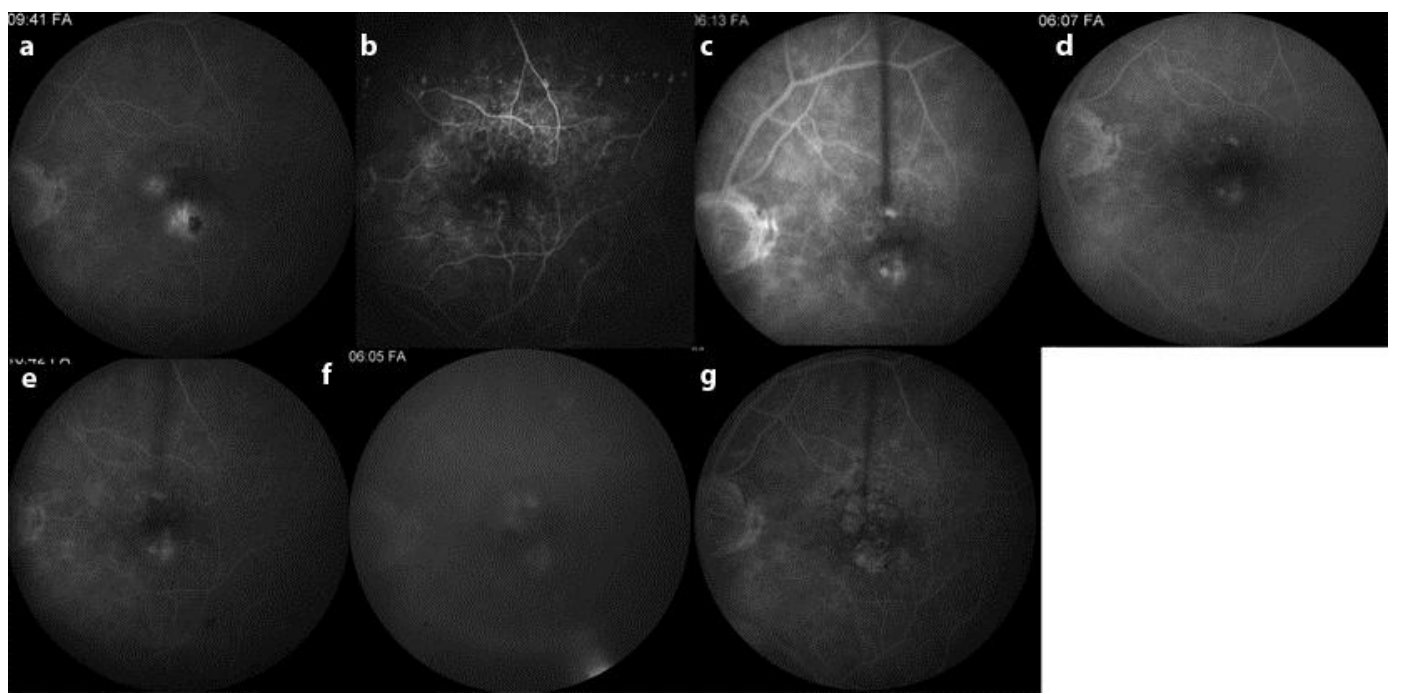

Fig. 1. Late-phase fluorescein angiograms from a patient treated with ranibizumab on an as needed basis over 47 months. The CNV lesion measured 2 disc areas at baseline (a). After the initial ranibizumab treatment, the CNV lesion decreased in size at month 1 (b) month 3 (c) and month 6 (d), maintained this size through month 9 (e), and increased at month 12 (f). At 47 months (g), there was no CNV activity but geographic atrophy had developed.

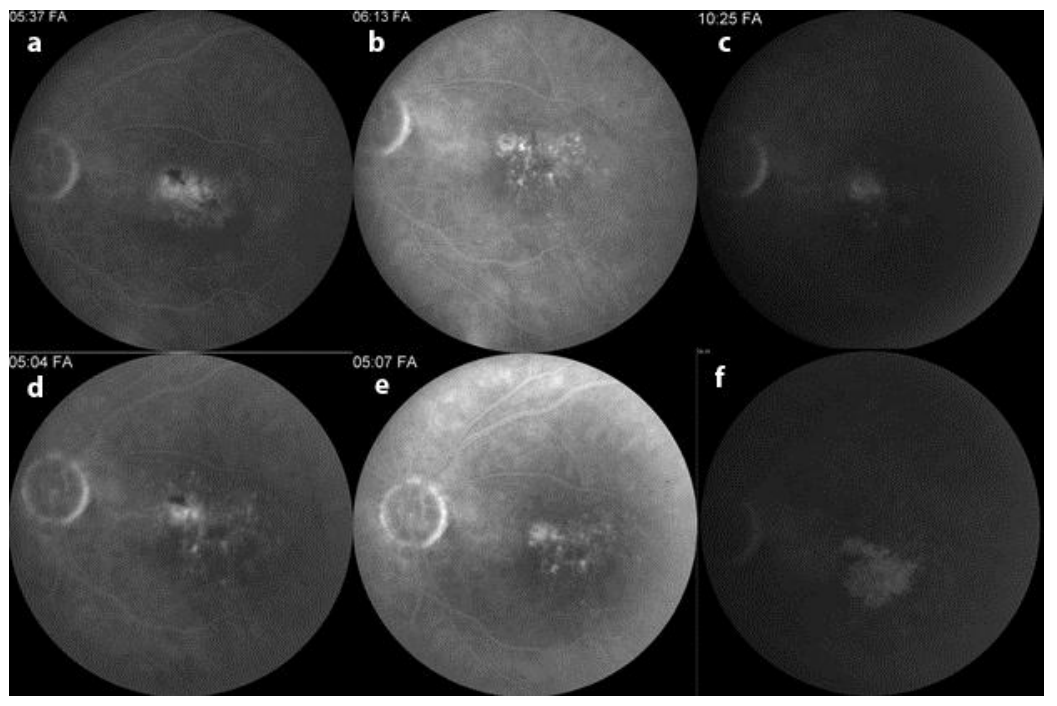

Fig. 2. Late-phase fluorescein angiograms from a patient treated with bevacizumab at baseline and treated with either bevacizumab or ranibizumab on an as needed basis for 57 months. The CNV lesion measured 1-2 disc areas at baseline (a). The lesion decreased in size slightly at month 1 (b) and then leaked more at month 5 (c), month 9 (d) and month 12 (e). Over 57 months, the CNV activity completely regressed though geographic atrophy had developed (f). 


\section{References}

1 Rosenfeld PJ, Brown DM, Heier JS, Boyer DS, Kaiser PK, Chung CY, Kim R: Ranibizumab for neovascular age-related macular degeneration. N Engl J Med 2006;355:1419-1431.

-2 Brown DM, Michels M, Kaiser PK, Heier JS, Sy JP, Ianchulev T: Ranibizumab versus verteporfin photodynamic therapy for neovascular age-related macular degeneration: two-year results of the ANCHOR study. Ophthalmology 2009;116:57-65.e5.

-3 Lalwani GA, Rosenfeld PJ, Fung AE, Dubovy SR, Michels S, Feuer WJ, Davis JL, Flynn HW, Esquiabro M: A variable-dosing regimen with intravitreal ranibizumab for neovascular age-related macular degeneration: year 2 of the PrONTO Study. Am J Ophthalmol 2009;148:43-58.e1.

4 Fung AE, Lalwani GA, Rosenfeld PJ, Dubovy SR, Michels S, Feuer WJ, Puliafito CA, Davis JL, Flynn HW, Esquiabro M: An optical coherence tomography-guided, variable dosing regimen with intravitreal ranibizumab (Lucentis) for neovascular age-related macular degeneration. Am J Ophthalmol 2007;143:566-583.

5 Martin DF, Maguire MG, Ying GS, Grunwald JE, Fine SL, Jaffe GJ: Ranibizumab and bevacizumab for neovascular age-related macular degeneration. N Engl J Med 2011;364:1897-1908.

-6 Sandhu SS, Talks SJ: Correlation of optical coherence tomography, with or without additional colour fundus photography, with stereo fundus fluorescein angiography in diagnosing choroidal neovascular membranes. Br J Ophthalmol 2005;89:967-970.

-7 Kotsolis AI, Killian FA, Ladas ID, Yannuzzi LA: Fluorescein angiography and optical coherence tomography concordance for choroidal neovascularisation in multifocal choroidtis. Br J Ophthalmol 2010;94:1506-1508.

8 Kaiser PK, Blodi BA, Shapiro H, Acharya NR: Angiographic and optical coherence tomographic results of the MARINA study of ranibizumab in neovascular age-related macular degeneration. Ophthalmology 2007;114:1868-1875.

-9 Khurana RN, Dupas B, Bressler NM: Agreement of time-domain and spectral-domain optical coherence tomography with fluorescein leakage from choroidal neovascularization. Ophthalmology 2010;117:1376-1380.

10 Rosenfeld PI, Shapiro H, Tuomi L, Webster M, Elledge J, Blodi B; MARINA and ANCHOR Study Groups: Characteristics of patients losing vision after 2 years of monthly dosing in the phase III ranibizumab clinical trials. Ophthalmology 2011;118:523-530. 